REVIEW ARTICLE

\title{
Central Sleep Apnea Syndromes - A Review
}

\section{Anuja Sharma}

Allina Medical Clinic, MN, USA

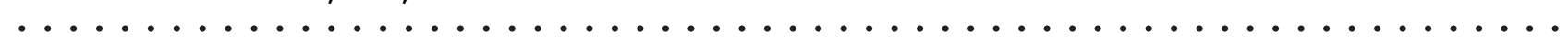

Indian J Sleep Med 2011 ; 6.4, 130-134

\section{Definition}

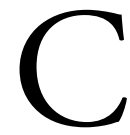
entral Sleep Apnea Syndrome (CSAS) is a disorder characterized by recurrent episodes of apnea during sleep, resulting from temporary suspension of ventilatory effort in association with symptoms.

The diagnosis is made by complete nocturnal polysomnograms (NPSG) as algorithms in portable monitors assume obstructive sleep apnea as the underlying etiology. During NPSG, absence of airflow in absence of respiratory effort characterizes central apneas. The hallmark is complete absence of ventilatory drive leading to lack of electromyographic activity in the respiratory muscles and absence of pleural pressure swings, measured by esophageal pressure measurements. Central and obstructive hypopneas are difficult to differentiate. Presence of "inspiratory flow limitation" seen in the nasal pressure transducer suggests an obstructive etiology. However, the esophageal balloon remains the "gold standard" for accurate detection and classification of hypopneas. The later technique is not used in routine clinical practice.

In general, central events are not seen in isolation. Most of these patients do have some element of obstruction.

Patients with predominantly Central Sleep Apnea (CSA)constitute less than $10 \%$ of persons with sleep apnea in most sleep laboratories. It does not represent a single entity or result from a single cause. Thus, all possible causes need to be considered while taking care of patients with CSA.

This article will not discuss central hypoventilation syndromes.

\section{Address for correspondence \\ Anuja Sharma \\ Allina Medical Clinic, MN, USA}

Indian Journal of Sleep Medicine (IJSM), Vol. 6, No. 4, 2011

\section{Pathophysiology}

Central apneas are associated with complete lack of electromyographic activity of the ventilatory muscles and thus represent a loss of inspiratory drive. Though the pathogenesis is not entirely clear, it is important to understand the concepts of control of breathing and loop gain theory.

\section{Control of breathing}

Metabolic control system- chemoreceptors (carotid body for hypoxia and carotid body plus medullary chemoreceptors for hypercapnia), vagally mediated intrapulmonary receptors, brainstem mechanisms (pre Botzinger Complex)

Behavioral control system- activities of normal living influence ventilation. Neural input to respiration comes from the forebrain

Wakefulness System- neural input is from the descending arousal system of respiratory pattern generators in brainstem or from nonrespiratory sensory mechanisms

Studies show that during sleep, especially NREM, breathing is controlled only by metabolic control system that is hypoxia and hypercapnia. Sleep abolishes wakefulness related excitatory input. Interestingly, information suggests that ventilatory responses to hypoxia and hypercapnia are reduced during NREM sleep and decrease further in REM sleep.

\section{Ventilatory control stability}

Abnormalities in the various components of theventilatory system can lead to ventilatory instability. This is best understood by the concept of "loop gain", a product of plant gain and controller gain. It is defined as the ventilatory response to a disturbance of ventilation. 
A "large" gain indicates an unstable system (prone to large swings in blood gases and ventilation) whereas a "small" gain indicates a stable system (with little or no ventilatory fluctuations in response to a disturbance). Also, the dynamic aspects of loop gain are important to the ventilatory stability. Thus, loop gain is not a single number but depends upon the duration of disturbance and the time it takes for the response to reach a steady state.

As noted, the respiration during NREM sleep is critically dependent on chemical influences, especially carbon dioxide tension $(\mathrm{PaCO} 2)$. Central apnea results if arterial $\mathrm{PaCo} 2$ is lowered below a highly sensitive "apneic threshold" (level of $\mathrm{PaCO} 2$ below which respirations cease). Thus, a variety of stimuli induce hyperpnoea (as in arousal from sleep), ventilatory overshoot leads to hypocapnia which induces central apnea (as the patient falls asleep again and the metabolic control takes over and the $\mathrm{PaCO} 2$ falls below the apneic threshold). The central apnea causes $\mathrm{PaCO} 2$ to rise, mild hypercapnia (4-6 mm $\mathrm{Hg}$ above eucapnia) restores rhythmic respiration, then ventilatory overshoot and hypocapnia leading to another central apnea. Clearly, a "large" loop gain will initiate and propagate the condition whereas "small" loop gain will stabilize it.

\section{Classification (International Classification of Sleep Disorders 2)}

1. Primary Central Sleep Apnea

2. Central Sleep Apnea due to cheyne stokes breathing pattern

3. Central Sleep Apnea due to High Altitude Periodic breathing

4. Central Sleep Apnea due to medical condition not Cheyne Stokes

5. Central Sleep Apnea due to drug or substance

6. Primary Sleep Apnea of Infancy

\section{Epidemiology}

The range of incidence cited in the literature is wide, depending upon the population studied, method used and threshold used to define it.

In 2005, American Academy of Sleep Medicine defined primary CSAS as the presence of five or more central events per hour of sleep in association with excessive daytime sleepiness, nocturnal arousals and awakenings, or awakenings with shortness of breath. Cheyne Stokes respirations were defined as at least 10 central apneas and hypopneas per hour, with a crescendodecrescendo tidal volume pattern, in a patient with a serious medical condition.

Studies of symptomatic primary CSA did not define any consistent epidemiologic trend as far as sex predominance is concerned. It was noted to be more common in middle aged to older adults.

Cheyne Stokes respirations (CSR) is noted in patients with congestive heart disease, particularly in men over 60 years of age. Studies indicate that almost $40 \%$ of patients with left ventricular ejection fractions less than $45 \%$ demonstrate such pattern of breathing.It is also noted in $10 \%$ of patients with strokes and also in chronic renal failure. The pattern and mechanism for the later two are less well studied.

Central Sleep Apnea due to Medical Condition not Cheyne Stokes is seen in people with lesions of the brainstem.

Central Sleep Apnea due to Drug or Substance is commonly seen in patients on methadone maintenance treatment and those on chronically narcotics.

\section{Diagnostic Evaluation}

Diagnosis involves complete NPSG with special attention to identifying inspiratory effort. The "gold" standard for the same is an esophageal balloon catheter recording intrathoracic pressure swings that reflect ventilatory effort. This is used in research. In clinical laboratories, complete absence of thoracoabdominal motion using respiratory inductive plethysmography (RIP) or strain gauges strongly suggests central event. Another method uses diaphragmatic electromyography, though difficult to obtain and not always reliable. Inspiratory flow limitation in nasal pressure transducer tracing suggests obstructive etiology. Pulse wave artifact in oronasal tracing suggests an open airway and thus, central etiology. In summary, it is difficult to distinguish central from obstructive hypopneas without noninvasive monitoring techniques. Thus, it is important to document central apneas in an attempt to definitively diagnose CSA. 


\section{Clinical presentation, Pathogenesis and Management}

\section{Primary (Idiopathic) Central Sleep Apnea}

It is characterized by recurrent episodes of cessation of respiration during sleep with no associated respiratory effort leading to sleep fragmentation and thus, excessive daytime sleepiness, nocturnal awakenings and insomnia. These generally occur at a frequency of 5 or more per hour, are 10 seconds or longer, occur at the onset of NREM sleep and less so during stable NREM, are less common in REM sleep, cause only mild oxygen desaturations. $\mathrm{PaCO} 2$ is generally less than $40 \mathrm{mmHg}$ during wakefulness. The exact mechanism remains unclear. Mechanisms suggested include high hypercapnic response (elevated loop gain). Long expiratory pause terminated by a large breath suggests a failure of the expiratory-inspiratory switch, possible influenced by chemoreceptors and other mechanisms (lung volume, chest wall mechanoreceptors, blood pressure).

In general, patients with primary CSA less commonly complain of excessive daytime sleepiness when compared with patients with OSA. They may have insomnia, restless sleep, or frequent awakenings through the night. The last may be accompanied by gasping or shortness of breath. The body habitus is normal. There is no evidence that these events cause any adverse cardiopulmonary consequences because significant hypoxia or hypercapnia is rare. Insomnia and nasal obstruction may exacerbate the situation.

A wide variety of medications have been attempted including acetazolamide, theophylline, medroxyprogesterone, naloxone, benzodazepines and clomipramine. These drugs have not been systematically studied. A recent review recommended acetazolamide as an option to treat primary CSAS. The same paper recommended use of zolpidem and triazolam. only in absence of risk factors for respiratory depression.

CPAP may be effective in some patients especially in those with an underlying obstructive pathology and reflex inhibition of respiration. It may also be helpful in primary CSA by virtue of increasing $\mathrm{pCO} 2$ to above apnea threshold. Others may benefit from bi-level ventilation with timed back up rate mode.

Supplemental oxygen or increasing concentration of $\mathrm{CO} 2$ in inspired air may have a beneficial effect in stabilizing breathing. But once again, larger trials are needed to address both safety and efficacy.

\section{Central Sleep Apnea due to Cheyne Stokes breathing pattern}

It is characterized by recurrent apneas, hypopneas or both alternating with prolonged hyperpneas in which tidal volume waxes and wanes in a crescendo-decrescendo pattern, seen primarily in NREM sleep leading to oxygen desaturation and sleep fragmentation. The prolonged waxing and waning of the hyperpnea with arousal most commonly seen at the peak of hyperpnea are characteristics of this breathing pattern and not seen in any other form of CSA.

The central apneas are a result of ventilatory control system instability (high loop gain). Thus, the chronic hyperventilation and consequent hypocapnia such that with onset of sleep the CO2 level is below the apnea threshold, arousals from sleep that cause increase in ventilation and the $\mathrm{CO} 2$ level drops below the arousal threshold and increased sensitivity of chemoreceptors. The crescendo-decrescendo pattern is secondary to increased sensitivity to chemoreceptors and prolonged circulatory delay secondary to decreased cardiac output. However, in animal experiments increasing circulation time in absence of cardiac disease did not precipitate this form of breathing. It is seen most commonly on transition from wakefulness to NREM 1 and 2, less so in NREM 3 or REM. Interestingly, periodic limb movements of sleep are seen in about $85 \%$ of patients with this.

It can be a cause of excessive daytime sleepiness, insomnia and paroxysmal nocturnal dyspnea. Studies have shown decreased survival rates in patients with CHF and CSR when compared with CHF alone, possibly secondary to a combination of increased hypoxia and catecholaminesurge. A large percentage of patients are asymptomatic.

Management should include aggressive optimization of CHF first using angiotensin converting enzyme inhibitors, beta blockers, digoxin therapy, aldosterone antagonism and diuretic therapy. CSR will frequently be eliminated in this case.

Oxygen therapy has been used to stabilize the breathing pattern. It is now recommended as a standard therapy based on data supporting improvement in AHI 
and left ventricular ejection fraction. Moreover, it is easily available. It is a safe option for patients who either refuse to or are unable to comply with use of CPAP. However, there is no data to support improvement in daytime symptoms.

Other options include useof methylxanthines. These drugs remain a theoretical alternative but are not generally used because of lack of complete efficacy in the short term, lack of documentation of long term efficacy and potential for significant side effects. Similarly, carbonic anhydrase inhibitors have been studied but are not in common use. These drugs work by lowering the $\mathrm{CO} 2$ threshold for apneas. However, clinical outcome data is lacking. These are considered as the last resort, that is after optimization of congestive heart failure and failure of CPAP. Close supervision is needed because of potential for adverse reactions.

CPAP reduces both cardiac preload, afterload, catecholamines, hypoxemia and arousals. Studies show improvement in left ventricular function, suppression of catecholamines and improvement in breathing patterns with CPAP therapy. However, the CANPAP study was published in 2005 with no benefit of CPAP in CSR.In fact, there was increase in mortality in the CPAP group initially compared with control group. The study was terminated prematurely. Also there was marked improvement in survival regardless of randomization, likely secondary to improvement in medical management over time. Lack of mortality benefit could have been secondary to suboptimal CPAP pressure. Posthoc analysis of the CANPAP trial database was published in 2007 suggested improved left ventricular ejection fraction and transplant free survival in patients whose AHI was corrected to less than 15 . The same review cited above recommends use of CPAP as the first line treatment. However, it should be able to normalize AHI.

Adaptive servoventilators, a new generation of positive pressure machines, work to dampen fluctuations in sleep disordered breathing by applying supplementary support during the apnea-hypopnea phase but only CPAP (or minimal support) during the hyperpnea phase with near complete resolution of CSR. Data indicates improvement in AHI and left ventricular ejection fraction along with better compliance in comparison with CPAP. Large scale trials and survival data are not available at this time. It is now recommended as a standard therapy for CSR. The cost of the device far exceeds that of CPAP and it is less widely available.

\section{Central Sleep Apnea due to Drug or Substance}

It is seen in $30-75 \%$ of patients on narcotics chronically, especially long acting narcotics, and is most commonly associated with methadone. The strongest predictor is dose. Such patients may also have other sleep related breathing abnormalities such as hypoventilation, obstructive sleep apnea and periodic breathing. The underlying pathology is felt to be respiratory depression induced by these drugs.

Treatment should include reduction in the dose of narcotics (as the disorder is dose dependent) and consideration of alternative drugs and therapies to control chronic pain. Alternatively, bi-level ventilation with fast back up rate or adaptive servoventilation may be beneficial. CPAP is ineffective. Data is extremely limited and best approach is yet to be determined.

\section{Summary}

CSA can be primary or secondary. Obstructive respiratory often occur in association with central events.

The pathogenesis remains unclear but is believed to be related to instability in ventilatory control. Patients develop cycles of apnea/hypopnea and hyperpnoea, ventilatory overshoot, hypocapnia, central apnea, hypercapnia, recurrent hyperpnoea.

CSA is uncommon but increased in prevalence amongst elderly, males, congestive heart failure, stroke, chronic renal failure and patients who take narcotics.

NPSG is necessary to make a diagnosis. It should demonstrate a predominance of central apneas. The events are mostly seen in awake to sleep transitions and NREM sleep and do not cause severe desaturations.

Symptoms may include disrupted sleep, insomnia, excessive daytime sleepiness and fatigue. In general, patients are less sleepy than patients with obstructive sleep apnea.

Treatment should be directed at treating the underlying cause such as congestive heart failure and use of narcotics.

Primary CSA may be treated with positive pressure therapy. Acetazolamide, zolpidem and triazolam remain distant options.

CSR may be treated with CPAP targeted to correct

Indian Journal of Sleep Medicine (IJSM), Vol. 6, No. 4, 2011 
AHI, adaptiveservo ventilators and possibly nocturnal oxygen. Acetazolamide and theophylline again remain distant options

\section{Suggested reading}

1. Kryger, Meir H., T. Roth, and William C. Dement. Principles and Practice of Sleep Medicine. Philadelphia, PA: Saunders/ Elsevier, 2011. Print.

2. American Academy of Sleep Medicine. The International Classification of Sleep Disorders., $2^{\text {nd }}$ ed:Diagnostic and Coding Manual. Westchester, Illinois:American Academy of Sleep Medicine, 2005. Print.

3. Eckert DJ, Jordan AS, Merchia P et al. Central sleep apnea: pathophysiology and treatment. Chest 2007; 131:595-607.

4. Malhotra A, Berry R, White D. Central sleep apnea. Clin Sleep Disord 2005;331-346.

5. Bradley TD, Logan AG, Kimoff TJ et al. Continuous positive airway pressure for central sleep apnea and heart failure. N Engl J Med 2005;353:2025-2033.

6. Arzt M, Floras JS, Logan AG, et al. Suppression of central sleep apnea by continuous positive airway pressure and transplant-free survival in heart failure: a post hoc analysis of the Canadian Continuous Positive Airway Pressure for Patients with Central Sleep Apnea and Heart Failure Trial (CANPAP). Circulation 2007; 115:31403142.
7. Sasayama s, Izumi T, Matsizaki $M$, et al. Improvement of quality of life with nocturnal oxygen therapy in heart failure patients with central sleep apnea, Circ J 2009; 73: 1255-628

8. Jahaveri S. Acetazolamide improves central sleep apnea and heart failure: a double blind, prospective study. Am J RespirCrit Care Med 2006;353:2025-2033.

9. Krachman S, Nugent T, Crocetti J, D'Alonzo $G$ et al. Effects of oxygen therapy on left ventricular function in patient with Cheyne-Stokes respiration and congestive heart failure. $J$ Clinc Sleep Med 2005; 1:271-6.

10. Javaheri S. Acetazolamide improves central sleep apnea in heart failure:a double-blind, prospective study. Am J RespirCrit Care Med2006; 173:234-7.

11. Walker JM, Farney RJ, Rhondeau SM et al. Chronic opioid use is a risk factor for developing central sleep apnea and ataxic breathing. J Clin Sleep Med 2007;3(5):455-461.

12. Farney RJ, Walker JM, Boyle KM, et al. Adaptive servoventilation (ASV) in patients with sleep disordered breathing associated with use of opioids. J Clin Sleep Med 2008; 4:311-319.

13. Allam JS, Olson EJ, Gay PJ, Timothy Morgenthaler. Efficacy of adaptive servoventilation in treatment of complex and central sleep apnea syndromes. Chest 2007; 132:1839-46.

14. Morgenthaler TI, Gay PJ, Gordon N, Brown LK. Adaptive servoventilation versus noninvasive positive pressure ventilation for central, mixed and complex sleep apnea syndromes. Sleep 2007;30:468-75.

15. Aurora RN, Chowdhuri S, Ramar K, Bista S, et al. The treatment of central sleep syndromes in adults: practice parameters with an evidence- based literature review and meta-analyses. Sleep 2012;35(1):17-40. 